\title{
Intra uterine insemination: Limitations in a tertiary care hospital
}

\author{
Rekha Rani', Maruti Sinha ${ }^{2}$, Ratnaboli Bhattacharya ${ }^{3}$, Ridhima Gupta ${ }^{4}$, Gurcharan Kaur ${ }^{5}$ \\ ${ }^{1}$ CMO, Family Planning, Kasturba Hospital, Delhi, ${ }^{2}$ Consultant Obs \& Gynaecology, Kasturba Hospital, Delhi, ${ }^{3}$ GDO Obs \& Gynaecology, \\ Kasturba Hospital, Delhi, ${ }^{4}$ Resident Physician, Charleston Medical Centre, West Virginia University, Charleston, West Virginia, ${ }^{5}$ Consultant and \\ Head of Department, Kasturba Hospital, Delhi
}

\section{A B S TR A C T}

Objective: Infertile couples need expert guidance to be able to choose an appropriate therapy relevant to their sub-fertility where one in seven couples have infertility. The present study has been designed to study the clinical effectiveness of IUI in enhancing pregnancy rates in cases of unexplained infertility and to compare it with other modalities of expectant treatment. We enumerate our experience with IUI at Kasturba Hospital with a special effort to assess any limitations in performing this procedure in tertiary care centres like ours. Methods: This was a prospective study with observational analysis of data on sub fertile couples who underwent $\mathrm{COH}$ and IUI as a part of the management protocol in our hospital- Kasturba Hospital, New Delhi. The period of study extended from 2007 to 2014. Infertility work up of all the registered couples was done on a standard investigation protocol. The male partner was also assessed by a detailed history and semen analysis. Results: Average females were less than 30 years of age at $48.1 \%$ Majority of couples were having primary infertility $(82.28 \%)$ whereas only $17.72 \%$ had secondary infertility. Pregnancy rates achieved with single IUI $(22.55 \%)$ was more than that achieved in double IUI $(11.11 \%)$. Pre rupture IUI resulted in higher pregnancy rates $(23.08 \%)$ than post rupture ones $(15.12 \%)$. Out of pregnancies that occurred from IUI cycles, term deliveries were $21.52 \%$ and there were $6.33 \%$ spontaneous miscarriages. Only $2.53 \%$ had twin pregnancies. Conclusion: In a resource deprived country like India where low per capita income make IVF-ET an unaffordable option to most of the patients, IUI has found wide acceptance with much lower costs. However the biggest limitation in offering IUI is that most of the tertiary care centres in India are yet to eqiup itself with standard IUI Laboratories to thus make Intra Uterine insemination widely available at affordably low cost.
Access this article online Website:

http://nepjol.info/index.php/AJMS DOI: 10.3126/ajms.v7i1.12614

\section{INTRODUCTION}

Infertile couples need expert guidance to be able to choose an appropriate therapy relevant to their sub-fertility, which demands evidence based review of different treatment options available with respect to their benefits and harm to patients \& clinicians. ${ }^{1}$ One in seven couples have infertility which remains unexplained as no abnormalities are detected in standard investigations for semen, patency of tubes and ovulation studies. $^{2}$

There is little role of expectant management in infertile patients and practitioners have over the years been relying heavily on empirical interventions which have not been rigorously evaluated previously. Controlled ovarian stimulation with Clomiphene Citrate and Intra Uterine Insemination is usually considered the first line of treatment in couples with unexplained infertility. 3,4

One of the reasons for the wide popularity of oral Clomiphene is the easy availability of the drug and its low cost thus making it very affordable. It is also considered clinically safe and thought to correct subtle ovulatory dysfunction. ${ }^{5}$ The cervical barrier can be overcome by Intra Uterine Insemination of the sperm and data suggests a threefold enhancement in pregnancy rates following IUI, 
with potential for further increase with concomitant use Gonadotrophins for superovulation. ${ }^{6}$ Superovulation with IUI is superior to unstimulated IUI. ${ }^{7}$

Drug regimens used in stimulated superovulation IUI cycles are similar to those used in IVF cycles. Close monitoring by means of TVS scans, estrogen concentration estimations allows control of number of follicles reaching maturity prior to induction of ovulation and insemination. Stimulated IUI has been used for a number of years as a treatment of subfertility but protocols and regimes vary between different IVF centres and clinicians. However in essence IUI involves artificial induction of ovulation combined with appropriately timed delivery of sperm into the uterine cavity. ${ }^{8}$ Though the technique of IUI remains same, several advances in type of stimulation protocols, gonadotrophins, sperm preparation techniques and USG monitoring have led to promising success rates with IUI. ${ }^{9}$ It is thus considered a viable treatment option for male factor infertility, cervical factor and unexplained infertility. ${ }^{10}$

Ovulation induction with clomiphene citrate or gonadotrophins (HMG/FSH) and intrauterine insemination (IUI) are often chosen before resorting to IVF. The appropriateness of starting with these low and intermediate intensity treatments is supported by evidence that CC/IUI increases cycle fecundity 2-3 fold and FSH/IUI increases cycle fecundity 3-5 fold over baseline chances of pregnancy. ${ }^{1}$

However, superovulation with intrauterine insemination is not a natural choice for treatment of unexplained infertility. ${ }^{8}$ OI/IUI has its own adverse effects which deserve attention particularly multiple pregnancies and ovarian hyperstimulation syndrome (OHSS). ${ }^{1}$ But practice guidelines published by RCOG suggest that IUI is an effective treatment. ${ }^{11} \mathrm{~A}$ multicentric RCT comparing intracervical insemination with IUI suggest FSH/IUI increased the likelihood of pregnancy. ${ }^{7}$

The utility of $\mathrm{COH} \&$ IUI as a primary interventional method for treatment of unexplained infertility is being widely debated in the western world and literature suggests that it appears to be as effective as IVF and should be offered to all patients as it is less invasive and requires fewer resources. Lately National Institute of Clinical Excellence (NICE) UK has revised the evidence of assessment and treatment of infertile couples and has recommended that IUI should be offered to couples prior to IVF. ${ }^{12}$

The present study has been designed to study the clinical effectiveness of IUI in enhancing pregnancy rates in cases of unexplained infertility and to compare it with other modalities of expectant treatment. We enumerate our experience with IUI at Kasturba Hospital with a special effort to assess any limitations in performing this procedure in a government setup like ours.

\section{MATERIALS AND METHODS}

This is a prospective study with observational analysis of data on subfertile couples who underwent $\mathrm{COH}$ and IUI as a part of the management protocol in our hospital- Kasturba Hospital, New Delhi. The period of study extended from 2007 to 2014.

Infertility work up of all the registered couples was done on a standard investigation protocol and included a detailed history with special emphasis on her medical history, a thorough clinical examination, general investigations, hormonal analysis, hysterosalpingography and ultrasonography. Wherever indicated diagnostic hysteroscopy and laparoscopy was also performed. The male partner was also assessed bya detailed history and semen analysis.

Women with tubo-ovarian masses, sever endometriosis, severe OATS, active tuberculosis and bilateral tubal block were excluded from this study. However patients were not excluded on age factor i.e. >35years. Couples included in the study were the ones with cervical factor infertility, minimal tubo-peritoneal endometriosis, mild degree of adnexal adhesions, anovulation, unexplained infertility and male factor subfertility.

A standard treatment protocol included controlled ovarian hyper stimulation by ovulation induction with clomiphene citrate with or without gonadotrophins. Sonographic folliculometry from D-8 onwards was routinely performed with administration of 5000IU of Inj Human Chorionic Gonadotrophin when follicular size of more than $18 \mathrm{~mm}$ was attained by LH surge.

Single IUI was performed between 36-44 hours of Inj. $\beta$-hCG and in a selected few where follicular rupture did not occur even after 24 hours. Semen preparation was by standard density gradient swim up technique by an accredited sperm lab. Luteal phase support with micronized progesterone was given to all patients on whom IUI was performed.

\section{OBSERVATIONS \& RESULTS}

79 subfertile couples were enrolled in our study, who underwent 120 IUI treatment cycles and there the observations were as follows:

It is evident from Table 1 that average females were less than 30 years of age at $48.1 \%$. Majority of couples were 
having primary infertility $(82.28 \%)$ whereas only $17.72 \%$ had secondary infertility.

In our study, predominant amongst all factors was anovulation (42 patients), where male factor as well as unexplained reasons were the cause in 37 patients each. Per cycle fecundity ( $\mathrm{n}=120$ cycles) according to the factor responsible for infertility, the highest success rate was observed in unexplained infertility $(29.73 \%)$. For male factor it was $10.8 \%$ and for anovulation it was $26.19 \%$. For tubo-peritoneal factors, the fecundity was $7.69 \%$ while none of the patients with combined factors conceived. Luteal phase defect or cervical factor was not found to be the cause of infertility in our study. The cycle fecundity was $20.83 \%$ and the pregnancy rate was $31.64 \%$ (Table 2 ).

Out of pregnancies that occurred from IUI cycles, term deliveries were $21.52 \%$ and there were $6.33 \%$ spontaneous miscarriages. Only $2.53 \%$ had twin pregnancies (Table 3 ).

When we compared various regimens used for ovulation induction for IUI we found that though the percentage

\begin{tabular}{|c|c|c|}
\hline Variable & No. of couples & $\%$ of couples \\
\hline \multicolumn{3}{|l|}{ Age of female } \\
\hline$<30$ yrs & 38 & 48.1 \\
\hline$>30 \mathrm{yrs}$ & 41 & 51.9 \\
\hline \multicolumn{3}{|c|}{ Duration of infertility } \\
\hline$<5$ yrs & 46 & 58.23 \\
\hline $5-10$ yrs & 27 & 34.18 \\
\hline$>10$ yrs & 6 & 7.59 \\
\hline \multicolumn{3}{|c|}{ Type of infertility } \\
\hline Primary & 65 & 82.28 \\
\hline Secondary & 14 & 17.72 \\
\hline
\end{tabular}

\begin{tabular}{|c|c|c|c|c|}
\hline Diagnosis & $\begin{array}{c}\text { No. of } \\
\text { patients } \\
(\mathrm{N}=79)\end{array}$ & $\begin{array}{l}\text { No. of } \\
\text { cycles } \\
(\mathrm{N}=120)\end{array}$ & Pregnancy & Fecundity \\
\hline Male factor & 18 & 37 & 4 & 10.8 \\
\hline Anovulation & 25 & 42 & 11 & 26.19 \\
\hline Unexplained Infertility & 32 & 37 & 11 & 29.73 \\
\hline Tubo-peritoneal factors & 11 & 13 & 1 & 7.69 \\
\hline Cervical Factor & 0 & 0 & 0 & 0 \\
\hline Luteal phase defect & 0 & 0 & 0 & 0 \\
\hline Others/Combined & 4 & 6 & 0 & 0 \\
\hline
\end{tabular}

\begin{tabular}{lcc}
\multicolumn{3}{l}{ Table 3: Outcome of pregnancy in IUI conceptions } \\
\hline Outcome variable & $\begin{array}{c}\text { No. of } \\
\text { females }\end{array}$ & $\begin{array}{c}\% \text { of } \\
\text { females }\end{array}$ \\
\hline Term live births & 17 & 21.52 \\
Preterm live births & 3 & 3.80 \\
IUD and stillbirth & 0 & 0 \\
Miscarriage and Ectopic pregnancy & 5 & 6.33 \\
Multiple pregnancy & 2 & 2.53 \\
\hline
\end{tabular}

of pregnancies achieved by low dose HMG either with $(10.05 \%)$ or without clomiphene (0) was low, pregnancy rate achieved with only clomiphene was higher at $40.91 \%$ which was very promising at a low cost (Table 4).

In our study we achieved maximum cycle fecundity $(39.47 \%)$ in the first cycle itself and collectively $58.52 \%$ pregnancy rate with first two attempts. None of the patients in our study underwent more than 4 attempts (Table 5).

Pregnancy rates achieved with single IUI $(22.55 \%)$ was more than that achieved in double IUI $(11.11 \%)$. Pre rupture IUI resulted in higher pregnancy rates $(23.08 \%)$ than post rupture ones $(15.12 \%)$ (Table 6).

Complications were not found subsequent to IUI procedures except in two patients who complained of abdominal cramps (Table 7).

\section{DISCUSSION}

What are the gains to a couple undergoing stimulated IUI for unexplained infertility? The process of stimulated IUI has the potential to 'bypasses' several influences on fertility. These include minor sperm abnormalities as sperm preparation increases normal motile sperms deposited in utero. Another advantage is due to avoidance of sperm- mucus interactions in the cervix. Timing of sperm delivery features in the success of stimulated IUI although it is likely that in a couple having regular intercourse, timing alone is not a significant factor in their failure to conceive. There may, in addition, be an advantage to some women

\begin{tabular}{lcc}
\multicolumn{3}{l}{ Table 4: Cycle fecundity by method of COH } \\
\hline Method & No. of patients & Pregnancies (\%) \\
\hline Clomiphene citrate & 44 & $18(40.91)$ \\
CC+HMG & 21 & $4(10.05)$ \\
CC+FSH & 9 & $2(22.22)$ \\
HMG & 1 & 0 \\
FSH & 2 & $1(50)$ \\
Others & 13 & $1(7.69)$ \\
\hline
\end{tabular}

\begin{tabular}{lcc}
\multicolumn{3}{l}{ Table 5: Cycle fecundity by attempt of IUI } \\
\hline No. of attempts of IUI & Pregnancy/cycle & Fecundity \\
\hline 1 & $15 / 38$ & 39.47 \\
2 & $8 / 42$ & 19.05 \\
3 & $2 / 27$ & 7.41 \\
4 & $0 / 4$ & 0 \\
\hline
\end{tabular}

\begin{tabular}{lcc}
\multicolumn{3}{|c}{ Table 6: Pregnancy rates achieved by timing of IUI } \\
\hline Timing of IUI & No. of cycles & Pregnancy achieved (\%) \\
\hline Single & 102 & $23(22.55)$ \\
Double & 18 & $2(11.11)$ \\
Pre rupture & 52 & $12(23.08)$ \\
Post rupture & 86 & $13(15.12)$ \\
\hline
\end{tabular}




\begin{tabular}{|c|c|c|c|c|c|}
\hline Sperm parameters & No. of cycle & No. of patients & No. of conceptions & $\%$ of conceptions & Conceptions/cycle \\
\hline \multicolumn{6}{|c|}{ Sperm count (in millions/ml) } \\
\hline$<5$ & 0 & 0 & 0 & 0 & 0 \\
\hline $5-10$ & 3 & 1 & 0 & 0 & 0 \\
\hline $10-20$ & 6 & 3 & 1 & 33.33 & 16.67 \\
\hline$>20$ & 111 & 75 & 24 & 32 & 21.62 \\
\hline \multicolumn{6}{|l|}{ Sperm motility (\%) } \\
\hline$\geq 50$ & 114 & 76 & 25 & 32.90 & 21.93 \\
\hline$<50$ & 6 & 3 & 0 & 0 & 0 \\
\hline
\end{tabular}

in the effects of the artificial regime on the endometrium and its function. ${ }^{?}$

The rationale put forward in support of $\mathrm{COH}$ and IUI is that ovarian stimulation corrects subtle, unpredictable ovulatory dysfunction with increase probability of conception if increased density of motile spermatozoon is placed close to multiple fertilizable oocytes. ${ }^{10}$

The concept of both IUI and IVF is based on the hypothesis that such procedures increase gamete proximity within the reproductive tract thus enhancing chances of conception. The universal preference for IUI has further increased following recommendations of National Institute of Clinical Excellence (NICE), UK which has revised the evidence for assessment and treatment of infertile couples and recommended that the IUI should be offered to couples with infertility because it is as effective as IVF, less invasive and requires fewer resources. ${ }^{11}$

In this study, an attempt to analyze couples with male and female subfertility undergoing $\mathrm{COH}$ and $\mathrm{OI}$ followed by IUI.

Several studies conducted in the past have reported varying degree of success with $\mathrm{COH} / \mathrm{IUI}$ in patients with unexplained infertility. Das et al achieved pregnancy rates of $7.47 \%,{ }^{9}$ comparable to $8.2 \%$ achieved by Steures et $\mathrm{al}^{13}$ and $9.2 \%$ reported by Iberico et al ${ }^{14}$ Higherconception rates of $18 \%$ were recorded by Agarwal et $\mathrm{al}^{15}$ from AIIMS which however was lower than the pregnancy rates of 20.83\% achieved in our set up. The pregnancy rate per couple was $31.64 \%$ in our study and was slightly more than $28.1 \%$ reported by Shibahara et al. ${ }^{16}$

Ovarian stimulation by Clomiphene citrate (CC) and IUI remains the first choice treatment for ovulatory dysfunction, unexplained infertility, endometriosis, male subfertility with pregnancy rate averaging $40.91 \%$ pregnancies. Pregnancy rates as high as $40.91 \%$ have been seen with $\mathrm{COH} / \mathrm{IUI}$ when it is the primary modality of treatment especially in cases of unexplained infertility, ovulatory dysfunction and endometriosis. Similar success rates in male factor sub fertility has also been reported with IUI. Owing to the negative influence of CC on endometrial thickness, the medication should be reduced to three days in patients with retarded endometrial growth confirmed on USG. ${ }^{17}$

Deshpande et $a 1^{18}$ in their study found pregnancy rates to be around $7.43 \%$.The preferred methods of ovulation induction include isolated use of Gonadotrophins like FSH \& HMG or in combination with GnRH agonist/ antagonist. Randomized trials are required to determine the dosage protocols with reference to low versus high dosage protocols, alternate versus daily dose regimes so as to optimize results. Current data suggests that low dose protocols are as effective in achieving good pregnancy rates and hence it is advisable that low dose regimes be used first. ${ }^{19}$

In our study we used HMG in a daily low dose of 75IU of HMG + HCG and in 19 patients higher doses of HMG were required with HCG. A very important limitation for the use of Gonadotrophins as a standard protocol in our hospital was the cost factor. On an average the patients attending our hospital belong to the lower socio economic class with severe financial constraints and are mostly unable to purchase Gonadotrophins. Few of them who did purchase the drug could not afford more than one to two cycles and unless these important medications are provided free of cost or at subsidized rates IUI success rates cannot be further enhanced in any setup despite it being a tertiary care centre.

The timing of insemination is also reported to influence the overall conception rate as per the accepted protocol. Insemination was ideally performed between 36-48 hours of HCG Injections anticipating follicular rupture during this period. Second inseminations were usually not performed except in cases where rupture failed to occur even after 48 hours of administration. However since the sperm preparation facility was not available in our hospital we had to rely on other Laboratories for the prepared semen and though standard laboratories were recruited our ability to monitor the semen preparation was limited. Also time of transportation of the prepared semen was dependant on the traffic movement of the area and many a times the sample had to be discarded due to extraordinary 
delay thus incurring financial losses to the patient and often led to abandonment of the cycle as patient could not afford a second sample. Although despite these constraints we had a comparable success rate with IUI, setting up of an internal IUI Laboratory in all tertiary hospitals would increase success rates and also decrease patient cost $\&$ difficulty.

Cycle fecundity by number of attempts was seen to be maximum in the first attempt. More than 4 cycles were not attempted. Studies by Khaleil et $\mathrm{a}^{20}$ have reported highest pregnancy rate in first treatment cycle.

Kerlin et al have observed that on an average 3.3 insemination cycles were required for each woman to become pregnant and few women conceived after five unsuccessful months of treatment. Age may also be an important factor in decreased fecundity in female and hence lower success rates in COH and IUI. ${ }^{21}$ Contrary to this Wiltbank et al reported an increase in success rateof IUI from $17 \%$ in $<3$ attempts to $33 \%$ in couples with $>3$ attempts. ${ }^{22}$

Ghosh et al have reported that women more than 30 years were half as likely to become pregnant as compared to women less than 30 years. ${ }^{23}$ However in our study, IUI was not refused to any patient less than 45 years.

Another factor influencing conceptions in couples undergoing $\mathrm{COH}$ and IUI could also be the duration of infertility. Iberico et al have in their studies mentioned a lower pregnancy rate where the duration of infertility was more than 3 years. ${ }^{14}$ In our study, maximum (58.23\%) couples had infertility for less than five years.

On analyzing pregnancy outcomes in conceptions through $\mathrm{COH}$ and IUI in our study, we found that there were $21.52 \%$ term live births, $3.80 \%$ preterm live births, $6.33 \%$ abortions as compared to Guzick et al, who reported $72 \%$ live births and $9.13 \%$ preterm live births ${ }^{7}$.

The fecundity rates could be further increased but unfortunately in developing countries like India, even a government teaching hospital like ours which is situated in Delhi and despite being a tertiary care hospital the resources are limited, there are severe financial constraints especially when finances are involved for setting up of an IUI Lab, purchase of instruments, technology up gradation and regular purchase of chemicals for semen preparation are concerned. Concerned official procedures involves a lot of file movement which takes years to be cleared. Also this being a resident based teaching hospital the IUI success rates are further limited by multiple performers and inevitable learning curve inherent to any such hospital which again is dependent upon supervised guidance of the senior faculty.

\section{CONCLUSION}

In a resource deprived country like India where low per capita income make IVF-ET an unaffordable option to most of the patients, IUI has found wide acceptance with much lower costs, much less invasiveness and nearly effective method of achieving successful pregnancies as IVF. In the Indian scenario, a patient must be given the option of $\mathrm{COH}$ and IUI for at least 6 cycles before referring her for costly IVF procedures which even in the best of hands and centers have a poor success rate of only $40-45 \%$. However more and more innovations need to be done to improve IUI outcomes and randomized controlled trials like flushing of cervix before IUI, endometrial scratching procedures etc. should be adopted as a method to increase success rates of insemination.

However the biggest limitation in offering IUI as a very viable alternative to IVF in management for Infertility as a first line of treatment is that most of the tertiary care centres in India are yet to eqiup itself with standard IUI Laboratories to thus make Intra Uterine insemination widely available at affordably low cost. In government set up even if the procedure is done free of cost, the drugs, reagent, semen preparation all entail huge cost to the patients thus making it widely unpopular.

\section{REFERENCES}

1. Hughes EG. 'Effective treatment' or 'not a natural choice'? Human Reproduction 2014;10:1093.

2. Templeton A. Infertility- epidemiology, aetiology, and effective management. Health Bull Edinb 1995; 53:294-298.

3. Royal College of Obstetricians and Gynaecologists. The management of infertility in secondary care: Evidence-based guidelines. No 3. London: RCOG, 1998.

4. Guzick DS, Sullivan MW, Adamson GD, Cedars ML, Falk RI, et al. Efficacy of treatment for unexplained infertility. Fertil Steril 1998; 70: 207-213.

5. Bhattacharya S, Harrild K, Mollison J, Wordswoth S, Tay C, et al. Clomifene Citrate or unstimulated intrauterine insemination compared with expectant management for unexplained infertility: Pragmatic randomized controlled trial. British Medical Journal 2008; 337: a716.

6. Hughes EG. The effectiveness of ovulation induction and intrauterine insemination in the treatment of persistent infertility: A meta-analysis. Hum Reprod 1997; 12:1865-1872.

7. Guzick DS, Carson SA, Coutifaris C, Overstreet JW, FactorLitvak $\mathrm{P}$, et al. Efficacy of super ovulation and intrauterine insemination in the treatment of infertility: National Cooperative Reproductive Medicine Network. N Engl J Med 1999; 340:177-183.

8. Stewart JA. Stimulated intrauterine insemination is not a natural choice for the treatment of unexplained subfertility. Should the guidelines be changed? Human Reproduction 2003; 18(5) 903-914.

9. Das V, Pandey A, Agarwal A, Mehrotra S and Pradeep Y. Intrauterine insemination experience in a government teaching hospital setup. J Obstet Gynecol India 2010; 60(4) 326-330. 
10. Gregoriou O, Pyrgiotis E, Konidaris S, Papadias C and Zourlas PA. Fallopian tube sperm perfusion has no advantage over intrauterine insemination when used in combination with ovarian stimulation for the treatment of unexplained infertility. Gynecol Obstet Invest 1995; 39(4):226-228.

11. RCOG The management of infertility in secondary care- Evidence-based guidelines. 1998; No.3. RCOG, London.

12. Deaton JL, Gibson M, Blackmer KM, Nakajima ST, Badger GJ and Brumsted JR. A randomized, controlled trial of clomiphene citrate and intrauterine insemination in couples with unexplained infertility or surgically corrected endometriosis. Fertil Steril 1990; 54(6):1083-1088.

13. Steures P, Van der Steeg JW and Mol BW. Prediction of an ongoing pregnancy after intrauterine insemination. Fertil Steril 2004; 82:45-51.

14. Iberico G, Vioque J, Auza N, Lozana JM, Roca M, Liacer J, et al. Analysis of factors influencing pregnancy rates in homologous intrauterine insemination. Fertil Steril 2004; 81:1308-1313.

15. Agarwal $S$ and Mittal $S$. A randomized prospective trial of intrauterine insemination versus timed intercourse in super ovulated cycle with Clomiphene. Indian J Med Res 2004; 120:519-522.

16. Shibahara $\mathrm{H}$, Obara $\mathrm{H}$, Ayustawati, Hirano $\mathrm{Y}$, Suzuki $\mathrm{T}$, Ohno A, et al. Prediction of pregnancy by intrauterine insemination using CASA estimates and strict criteria in patients with male factor infertility. Int J Androl 2004; 27:63-68.

17. Krzysztof L. Optimizing stimulation protocols In: Allahabadia GN Merchant R. editors. Contemporary perspective in assisted reproductive technology. India: Reed Elsevier; 205:10-7

18. Deshpande SA and Deshpande AB. Intrauterine insemination an experience in rural population. Int J Med Res Health Sci 2013; 2(3):341-349.

19. Kabli N, Sylvestre C, Tulandi T and Buckett W. Comparison of daily and alternate day recombinant $\mathrm{FSH}$ stimulation protocol for IUI. Fertil Steril 209; 91(4):141-144.

20. Khalil MR, Rasmussen PE, Erb K, Laursen SB, Rex S, et al. Homologous intrauterine insemination. An evaluation of prognostic factors based on a review of 2473 cycles. Acta Obstet Gynecol Scand 2001; 80:74-81.

21. Kerin JF, Kirby C, Peek J, Jeffrey R, Warnes GM, Matthews CD, et al. Improved conception rate after intrauterine insemination of washed spermatozoa from men with poor quality semen. Lancet 1984;1(8376):533-535.

22. Wiltbank MC, Kosasa $S$ and Rogers B. Treatment of infertile patients by intrauterine insemination of washed spermatozoa. Andrologia 1985; 17:22-30.

23. Ghosh C, Buck G, Priore R, Wacktawski-Wende J and Severino M. Follicular response and pregnancy among infertile women undergoing ovulation induction and intrauterine insemination. Fertil Steril 2003; 80(2):328-335.

Authors Contribution:

RR - Concept, Design, Definition of Intellectual Content, Literature search, Clinical studies, Data acquisition, Data analysis, Manuscript preparation;

MS - Concept, Design, Definition of Intellectual Content, Literature search, Clinical studies, Data acquisition, Data analysis, Manuscript preparation, Manuscript Editing; RB - Literature search, Clinical studies, Data analysis; RG - Manuscript editing; GK - Manuscript editing, Manuscript review.

Source of Support: Nil, Conflict of Interest: None declared. 